\title{
1508
}

\section{A comprehensive guide to pulmonary mesenchymal populations in the healthy and IPF lung.}

Idiopathic pulmonary fibrosis, Genomics, Personalised medicine

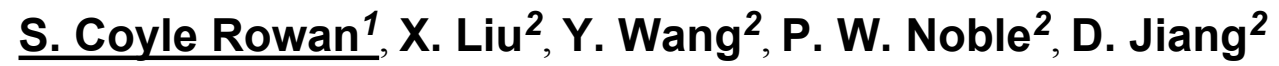

${ }^{1}$ University College Dublin - Dublin (Ireland), ${ }^{2}$ Cedars-Sinai Medical Centre - Los Angeles (USA)

\section{Background}

Exuberant tissue repair and extracellular matrix (ECM) deposition by pulmonary mesenchymal cells, notably fibroblasts, is believed to be central to the pathogenesis of fibrotic lung diseases like idiopathic pulmonary fibrosis (IPF). Few studies to date have utilised advanced omic technologies, like single cell RNA sequencing (scRNA-seq), to solely investigate these diverse and poorly described cell types.

\section{Aims and Objectives}

As there is no consensus on the mesenchymal subtypes present in the lung we performed a comprehensive analysis of pulmonary mesenchymal cells to identify all molecularly distinct subtypes, validate the efficacy of commonly used markers, identify new ones, and address longstanding controversies in the literature.

\section{Methods}

We undertook scRNA-seq and associated analyses on mesenchymal cells from healthy $(n=34)$ donors and IPF $(n=33)$ patients' lungs with comparative analyses in the murine lung (embryonic, post-natal, adult, aged, healthy vs. fibrotic).

\section{Results}

We identified, and resolved the transcriptome of; lipofibroblasts, myofibroblasts, smooth muscle cells, pericytes, mesothelial cells, a novel population delineated by Early B-cell Factor 1 expression and an "intermediate" subtype. Commonly reported markers, like ACTA2 or PLIN2, were found to be suboptimal. We identified conserved, novel, markers and transcription factors for all subtypes. These data suggest all mesenchymal subtypes contribute to ECM deposition in the IPF lung. We found no evidence of transdifferentiation between subtypes, nor an increase in myofibroblasts in the fibrotic lung. 


\section{Conclusions}

This study provides a basis for interrogating each mesenchymal subype and enhances our understanding of the contribution of these cell types to lung development, lung repair, and pulmonary fibrosis. 